\title{
Fast sensitivity-based optimal trajectory updates for descent operations subject to time constraints
}

\author{
Ramon Dalmau and Xavier Prats \\ Department of Physics - Aeronautics Division \\ Technical University of Catalonia - BarcelonaTECH \\ Castelldefels, Barcelona, Spain
}

\author{
Brian Baxley \\ Crew Systems and Aviation Operations Branch \\ NASA Langley Research Center (LaRC) \\ Hampton, VA, USA
}

\begin{abstract}
The ability to meet a controlled time of arrival while also flying a continuous descent operation will enable environmentally friendly and fuel efficient descent operations while simultaneously maintaining airport throughput. Previous work showed that guidance strategies based on a frequent recalculation of the optimal trajectory during the descent result in excellent environmental impact mitigation figures while meeting operational constraints in the presence of modelling errors. However, the time lag of recalculating the trajectory using traditional optimisation algorithms could lead to performance degradation and stability issues. This paper proposes an alternative strategy, which allows for fast updates of the optimal trajectory based on parametric sensitivities. Promising results show that the performance of this method is comparable to that of instantaneously recalculating the optimal descent trajectory at each time sample.
\end{abstract}

\section{INTRODUCTION}

Continuous descent operations (CDO) with controlled times of arrival (CTA) at one or several metering fixes could enable more environmentally friendly procedures without compromising capacity. This type of flight operation requires flight management systems (FMS) not only able to compute an optimal trajectory satisfying CTAs, but also to safely and efficiently guide the aircraft during the execution of the descent such that these time constraints are successfully satisfied.

The computation of the optimal descent trajectory can be formulated as an optimal control problem [1], in which a given cost function (e.g, fuel consumption) is to be minimised while satisfying a set of constraints. State-of-the-art FMS compute the optimal descent trajectory before starting the descent. Then, this initial trajectory is "frozen" and the guidance system uses different strategies, which depend on the FMS manufacturer, to execute it. However, the initial trajectory shows only what can be achieved given perfect knowledge of the parameters associated with the aircraft performance and weather models. When the parameters used by the FMS do not match reality, the initial trajectory is no longer the most optimal for the actual conditions, and some operational constraints may be violated if errors are not actively nullified.

In previous work [2], the performance of various guidance strategies in the time and energy managed operations (TEMO) concept [3] were compared using a high-fidelity flight simulator, in particular environmental impact mitigation and the ability to meet operational constraints. Several descents subject to CTAs were simulated including errors in the parameters of the weather and aircraft performance models used by the FMS. Results showed that non-linear model predictive control (NMPC) [4], a guidance strategy based on a frequent update of the optimal trajectory during the execution of the descent, is very robust in terms of correcting energy (speed and altitude) and time deviations, providing at the same time acceptable fuel consumption and noise nuisance figures. Furthermore, other research [5] has recently demonstrated the feasibility of using NMPC to achieve precise spacing between aircraft, the objective of interval management (IM) operations.

Traditional NMPC strategies update the optimal trajectory by solving a non-linear programming (NLP) optimisation problem. Ideally, the trajectory is updated instantaneously right after measuring the actual state of the aircraft at each time sample. In practical applications, however, solving the NLP problem may take significant time, leading to potential stability issues and degrading the performance of the operation [6]. In order to reduce the execution time, educated simplifications in the models can be used [7], at the expense of reducing the accuracy of the solution. Other NMPC implementations compensate for computational delay by starting the optimisation in advance, setting the initial conditions of the new trajectory to the predicted state of the aircraft at a look-ahead time equal to the estimated execution time. However, the unpredictability of the execution time still remains a critical issue [8].

An alternative method widely used in process industries, such as chemical manufacturing, consists of computing fast trajectory updates in the neighborhood of the active optimal trajectory using the theory of neighboring extremals [9]. Parametric sensitivities are obtained by linearisation of the necessary conditions of optimality to rapidly update the optimal trajectory for small perturbations in the model parameters. This strategy, known as sensitivity-based NMPC (SbNMPC) [10], [11], [12], reduces the execution time while ensuring that operational constraints are satisfied and that the cost is minimised.

In this paper, the SbNMPC strategy is implemented to guide aircraft during a CDOs subject to CTAs, and several descents are simulated with intentional errors in the parameters used by the FMS to describe the wind profile. Then, the performance of SbNMPC in terms of fuel consumption and ability to satisfy operational constraints is compared with those of the openloop solution and the ideal NMPC (INMPC)[6], which ideally updates the optimal descent trajectory without delay. 


\section{BACKGROUND}

Non-linear model predictive control (NMPC) was introduced to the process industry in the 1970's. This guidance strategy is based on the solution, at each time sample, of an optimal control problem over a future time horizon [4]. The resulting optimal control is applied only until the next time sample, when the optimal control problem is solved again.

Typical NMPC applications consider a fixed-length time horizon, which advances an interval sample at each recalculation. However, when the system has to reach a certain state in a fixed amount of time, a shrinking horizon is often preferred. Using this strategy, the length of the horizon is not fixed but decreases by one interval sample at each time sample.

In Section II-A the discrete-time optimal control problem for the shrinking horizon NMPC is formulated. Section II-B shows two alternatives to update the optimal solution when using the shrinking horizon NMPC strategy. Finally, Section II-C presents the working principle of two NMPC variants.

\section{A. The shrinking horizon NMPC optimal control problem}

Let us discretise a fixed time interval $\left[t_{0}, t_{f}\right]$ into $N+1$ equidistant time samples $\left[t_{0}, t_{1}, \ldots, t_{N}\right]$. The optimal control problem starting at $t_{i}, i=0, \ldots, N-1$ and minimising a cost function $f^{i}$ in the horizon $\left[t_{i}, t_{i+1}, \ldots, t_{N}\right]$ is formulated as:

$$
\begin{array}{ll}
\min _{\substack{\boldsymbol{x}_{k}, \boldsymbol{u}_{k} \\
k=i, \ldots, N}} & f^{i}:=\phi\left(\boldsymbol{x}_{N}\right)+\sum_{k=i}^{N-1} \ell_{k}\left(\boldsymbol{x}_{k}, \boldsymbol{u}_{k}, \boldsymbol{d}\right) \\
\mathrm{s.t} & \boldsymbol{x}_{i}=\boldsymbol{\chi}_{i} \\
& \boldsymbol{x}_{k+1}=\boldsymbol{f}_{k}\left(\boldsymbol{x}_{k}, \boldsymbol{u}_{k}, \boldsymbol{d}\right) ; k=i, \ldots, N-1 \\
& \boldsymbol{h}_{k}\left(\boldsymbol{x}_{k}, \boldsymbol{u}_{k}, \boldsymbol{d}\right) \geq 0 ; k=i, \ldots, N-1 \\
& \boldsymbol{\psi}\left(\boldsymbol{x}_{N}, \boldsymbol{d}\right) \geq 0
\end{array}
$$

where $\boldsymbol{x} \in \mathbb{R}^{n_{x}}$ is the state vector, with initial conditions $\chi_{i}$; $\boldsymbol{u} \in \mathbb{R}^{n_{u}}$ is the control vector; and the vector $\boldsymbol{d} \in \mathbb{R}^{n_{d}}$ includes all the parameters of the model. In this formulation, $\ell: \mathbb{R}^{n_{x}} \times$ $\mathbb{R}^{n_{u}} \times \mathbb{R}^{n_{d}} \rightarrow \mathbb{R}$ is the stage cost, and $\phi: \mathbb{R}^{n_{x}} \rightarrow \mathbb{R}$ is the terminal cost. The dynamics of the state vector are expressed by a set of non-linear equations $f: \mathbb{R}^{n_{x}} \times \mathbb{R}^{n_{u}} \times \mathbb{R}^{n_{d}} \rightarrow \mathbb{R}^{n_{x}}$; $\boldsymbol{h}: \mathbb{R}^{n_{x}} \times \mathbb{R}^{n_{u}} \times \mathbb{R}^{n_{d}} \rightarrow \mathbb{R}^{n_{h}}$ and $\boldsymbol{\psi}: \mathbb{R}^{n_{x}} \rightarrow \mathbb{R}^{n_{\psi}}$ represent applicable path and terminal constraints, respectively.

\section{B. Optimal control solution updates}

In a generic NMPC, the optimal trajectory obtained from (1) is updated at each $t_{i}$, given the measured current state $\chi_{i}$ and the estimated parameters $\boldsymbol{d}$. Then, the resulting optimal control $\boldsymbol{u}_{i}$ is applied and the horizon shrinks one interval sample.

There exist two alternatives to optimally update the trajectory at each $t_{i}$. Section II-B1 presents the full solution method; Section II-B2 shows the sensitivity-based approach.

1) Full solution updates (including initial solution at $t_{0}$ ): The optimal trajectory can be updated by formulating the discrete-time optimal control problem (1) as a parametric nonlinear programming (NLP) problem, which can be solved by means of standard solvers. The formulation of the NLP is:

$$
\begin{array}{ll}
\min _{\boldsymbol{z}^{i}} & f^{i}\left(\boldsymbol{z}^{i}, \boldsymbol{p}_{i}\right) \\
\text { s.t } & \boldsymbol{g}^{i}\left(\boldsymbol{z}^{i}, \boldsymbol{p}_{i}\right) \geq 0
\end{array}
$$

where the following definitions have been considered:

$$
\begin{aligned}
\boldsymbol{z}^{i} & :=\left[\boldsymbol{u}_{i}^{T}, \boldsymbol{x}_{i}^{T}, \boldsymbol{u}_{i+1}^{T}, \boldsymbol{x}_{i+1}^{T}, \ldots, \boldsymbol{u}_{N}^{T}, \boldsymbol{x}_{N}^{T}\right]^{T} \\
\boldsymbol{g}^{i} & :=\left[\boldsymbol{f}_{i}^{T}, \boldsymbol{h}_{i}^{T}, \boldsymbol{f}_{i+1}^{T}, \boldsymbol{h}_{i+1}^{T}, \ldots, \boldsymbol{f}_{N-1}^{T}, \boldsymbol{h}_{N-1}^{T}, \boldsymbol{\psi}^{T}\right]^{T} \\
\boldsymbol{p}_{i} & :=\left[\boldsymbol{\chi}_{i}^{T}, \boldsymbol{d}^{T}\right]^{T}
\end{aligned}
$$

and $\boldsymbol{z}^{i}$ the vector of primal variables (including both discretised states and controls); $\boldsymbol{g}^{i}$ is the vector of constraints; and $\boldsymbol{p}_{i}$ is composed of both current state at $t_{i}$ and model parameters.

Note that in this paper a direct collocation approach has been assumed to transform the original optimal control problem into a NLP. Direct collocation methods discretise both states and controls at a set of points. The discretised states and controls become the primal variables of the NLP problem [13]. However, the algorithms proposed in this paper could be easily adapted to other methods such as direct multiple shooting.

The Lagrangian function associated to problem (2) is:

$$
L^{i}\left(\boldsymbol{z}^{i}, \boldsymbol{p}_{i}, \boldsymbol{\lambda}^{i}\right):=f^{i}\left(\boldsymbol{z}^{i}, \boldsymbol{p}_{i}\right)+\boldsymbol{\lambda}^{i T} \boldsymbol{g}^{i}\left(\boldsymbol{z}^{i}, \boldsymbol{p}_{i}\right)
$$

where $\boldsymbol{\lambda}^{i} \in \mathbb{R}^{n} \boldsymbol{g}^{i}$ is the Lagrange multipliers (or dual variables) vector paired up with the constraints $\boldsymbol{g}^{i}$, being $n_{\boldsymbol{g}^{i}}$ the number of elements in $\boldsymbol{g}^{i}$. At the optimal solution, the pairs of constraints and dual variables are divided into two complementary sets: the active set $\mathcal{G}_{a c}^{i}\left(\boldsymbol{g}^{i}, \boldsymbol{\lambda}^{i}\right)$ and the inactive set $\mathcal{G}_{i n}^{i}\left(\boldsymbol{g}^{i}, \boldsymbol{\lambda}^{i}\right)$ [11]. An optimal primal-dual pair $\left(\boldsymbol{z}^{i *}, \boldsymbol{\lambda}^{i *}\right)$ satisfies the first-order necessary conditions of optimality (also known as Karush-Kuhn-Tucker or KKT conditions) if [14]:

$$
\begin{aligned}
L_{\boldsymbol{z}}^{i}\left(\boldsymbol{z}^{i *}, \boldsymbol{p}_{i}, \boldsymbol{\lambda}^{i *}\right) & =f_{\boldsymbol{z}}^{i}\left(\boldsymbol{z}^{i *}, \boldsymbol{p}_{i}\right)+\boldsymbol{\lambda}^{i * T} \boldsymbol{g}_{\boldsymbol{z}}^{i}\left(\boldsymbol{z}^{i *}, \boldsymbol{p}_{i}\right)=0 \\
g\left(\boldsymbol{z}^{i *}, \boldsymbol{p}_{i}\right) & =0 ; \lambda^{*}>0 ; \forall(g, \lambda) \in \mathcal{G}_{a c}^{i} \\
g\left(\boldsymbol{z}^{i *}, \boldsymbol{p}_{i}\right) & >0 ; \lambda^{*}=0 ; \forall(g, \lambda) \in \mathcal{G}_{i n}^{i}
\end{aligned}
$$

where $(\cdot) \boldsymbol{z}$ and $(\cdot)^{*}$ denote $\mathrm{d}(\cdot) / \mathrm{d} \boldsymbol{z}$ and "optimal", respectively; and $(\cdot)^{T}$ represents the transpose of $(\cdot)$. It can be stated that $\mathcal{P}_{N-i}$ represents any NLP algorithm that provides the optimal primal-dual solution as a function of the parameters:

$$
\left[\boldsymbol{z}^{i *}, \boldsymbol{\lambda}^{i *}\right]=\mathcal{P}_{N-i}\left(\boldsymbol{p}_{i}\right)
$$

Note that the parameters for which parametric sensitivities will be computed in the next section include both initial state and model parameters. In addition, by definition of (5), the optimal primal-dual solution is an implicit functions of $\boldsymbol{p}_{i}$.

In this paper, $\mathcal{P}_{N-i}$ is formulated in CasADi [15], a symbolic framework for automatic differentiation and numeric non-linear optimisation, and solved by using the sequential quadratic programming (SQP) algorithm implemented by SNOPT (Sparse Non-linear OPTimiser) NLP solver [16]. 
2) Sensitivity-based updates: If the parameters $\boldsymbol{p}$ vector changes slowly from one time sample to the next, parametric sensitivities at the active optimal solution can be used to rapidly update the optimal trajectory for a perturbation $\Delta p$.

The parametric sensitivities of the primal and dual variables of the NLP with respect to the parameters vector at $t_{i}\left(\boldsymbol{z}_{\boldsymbol{p}}^{i}\right.$ and $\lambda_{\boldsymbol{p}}^{i}$, respectively) can be obtained by differentiating the KKT condition (4) at the active optimal solution $\left[z^{i *}, \lambda^{i *}\right]$ :

$$
\left[\begin{array}{cc}
L_{\boldsymbol{z} \boldsymbol{z}}^{i *} & -\boldsymbol{g}_{\boldsymbol{z}}^{i * T} \\
\boldsymbol{g}_{\boldsymbol{z}}^{i *} & 0
\end{array}\right]\left[\begin{array}{c}
\boldsymbol{z}_{\boldsymbol{p}}^{i} \\
\boldsymbol{\lambda}_{\boldsymbol{p}}^{i}
\end{array}\right]=-\left[\begin{array}{c}
L_{\boldsymbol{z} \boldsymbol{p}}^{i *} \\
\boldsymbol{g}_{\boldsymbol{p}}^{i *}
\end{array}\right]
$$

Being consistent with the notation, $(\cdot)^{i *}=(\cdot)\left(\boldsymbol{z}^{i *}, \boldsymbol{\lambda}^{i *}, \boldsymbol{p}_{i}\right)$. The linear system (6) can be solved for $\boldsymbol{z}_{\boldsymbol{p}}^{i}$ and $\boldsymbol{\lambda}_{\boldsymbol{p}}^{i}$, allowing to update the optimal solution using a simple first-order Taylor approximation as follows:

$$
\begin{aligned}
\boldsymbol{z}^{i *}\left(\boldsymbol{p}_{i}+\Delta \boldsymbol{p}_{i}\right) & =\boldsymbol{z}^{i *}\left(\boldsymbol{p}_{i}\right)+\boldsymbol{z}_{\boldsymbol{p}}^{i} \Delta \boldsymbol{p}_{i} \\
\lambda^{i *}\left(\boldsymbol{p}_{i}+\Delta \boldsymbol{p}_{i}\right) & =\boldsymbol{\lambda}^{i *}\left(\boldsymbol{p}_{i}\right)+\boldsymbol{\lambda}_{\boldsymbol{p}}^{i} \Delta \boldsymbol{p}_{i}
\end{aligned}
$$

where $\Delta \boldsymbol{p}_{i}$ is the perturbation in the parameters vector at $t_{i}$. Unfortunately, this fast and convenient parametric sensitivity update can only be used if the set of active constraints does not change after the perturbation [11]. In practical NMPC applications, however, constraints in $\mathcal{G}_{a c}^{i}$ may become inactive, or constraints in $\mathcal{G}_{i n}^{i}$ may become active when $\boldsymbol{p}_{i}$ is perturbed.

An interesting approach that accounts for active set changes after a perturbation in the NLP parameters vector was suggested by [17]. This approach consists of reformulating Eq. (6) as a quadratic programming (QP) optimisation problem:

$$
\begin{array}{ll}
\min _{\Delta \boldsymbol{z}^{i}} & \frac{1}{2} \Delta \boldsymbol{z}^{i T} L_{\boldsymbol{z} \boldsymbol{z}}^{i *} \Delta \boldsymbol{z}^{i}+\Delta \boldsymbol{p}_{i}^{T} L_{\boldsymbol{z} \boldsymbol{p}}^{i *} \Delta \boldsymbol{z}^{i}+f_{\boldsymbol{z}}^{i * T} \Delta \boldsymbol{z}^{i} \\
\text { s.t } & \boldsymbol{g}^{i *}+\boldsymbol{g}_{\boldsymbol{z}}^{i *} \Delta \boldsymbol{z}^{i}+\boldsymbol{g}_{\boldsymbol{p}}^{i *} \Delta \boldsymbol{p}_{i} \geq 0
\end{array}
$$

For the sake of convenience, the following set of first and second-order NLP functions sensitivities are defined as $\mathcal{F}^{i *}:=\left\{L_{\boldsymbol{z} \boldsymbol{z}}^{i *}, L_{\boldsymbol{z} \boldsymbol{p}}^{i *}, f_{\boldsymbol{z}}^{i * T}, \boldsymbol{g}_{\boldsymbol{z}}^{i *}, \boldsymbol{g}_{\boldsymbol{p}}^{i *}\right\}$. Here, $\mathcal{Q}_{N-i}$ represents any $\mathrm{QP}$ algorithm which provides optimal primal variables perturbation $\Delta \boldsymbol{z}^{i *}$ and dual variables $\lambda^{i *}$ as a function of $\Delta \boldsymbol{p}_{i}$ :

$$
\left[\Delta \boldsymbol{z}^{i *}, \boldsymbol{\lambda}^{i *}\right]=\mathcal{Q}_{N-i}\left(\Delta \boldsymbol{p}_{i}\right)
$$

The dual variables of the optimal solution computed with the unperturbed parameters vector (i.e., $\boldsymbol{\lambda}^{i *}\left(\boldsymbol{p}_{i}\right)$ ) are updated with those obtained from solving $\mathcal{Q}_{N-i}$, while the primal variables and parameters vector are updated as follows:

$$
\begin{aligned}
\boldsymbol{z}^{i *}\left(\boldsymbol{p}_{i}+\Delta \boldsymbol{p}_{i}\right) & =\boldsymbol{z}^{i *}\left(\boldsymbol{p}_{i}\right)+\Delta \boldsymbol{z}^{i *}\left(\Delta \boldsymbol{p}_{i}\right) \\
\boldsymbol{p}_{i} & =\boldsymbol{p}_{i}+\Delta \boldsymbol{p}_{i}
\end{aligned}
$$

The first-order update (10) will be accurate only for small $\Delta \boldsymbol{p}_{i}$. For large perturbations, the new solution must to be analysed to verify that the KKT conditions are still satisfied. This is accomplished by computing the error in the Lagrange sensitivity $\epsilon_{\text {opt }}$ and the non-linear constraint infeasibility $\epsilon_{\text {inf }}$ at the updated pair of primal-dual variables $\left[\boldsymbol{z}^{i *}, \boldsymbol{\lambda}^{i *}\right]$ and $\boldsymbol{p}_{i}$ :

$$
\epsilon_{\text {opt }}=\frac{\left\|L_{\boldsymbol{z}}^{i *}\right\|_{\infty}}{\left\|\boldsymbol{\lambda}^{i *}\right\|_{2}+1}, \epsilon_{\text {infs }}=\frac{\left\|\boldsymbol{g}^{i *}\right\|_{\infty}}{\left\|\boldsymbol{z}^{i *}\right\|_{2}+1}
$$

If these metrics were higher than a pre-defined threshold, $\mathcal{F}^{i *}$ would be updated with the new $\left[\boldsymbol{z}^{i *}, \lambda^{i *}\right]$ solution and $\boldsymbol{p}_{i}$, and further iterations of $\mathcal{Q}_{N-i}$ would be triggered by setting $\Delta \boldsymbol{p}_{i}=0$ until satisfying feasibility and optimality criteria.

\section{NMPC guidance strategies}

Depending on which of the methods presented in Section II-B is adopted to update the optimal trajectory, the following two NMPC variants can be defined:

1) Ideal NMPC (INMPC): In an ideal case, problem $\mathcal{P}_{N-i}$ is solved at each $t_{i}$, as soon as the parameter vector $\boldsymbol{p}_{i}$ is measured or estimated. Then, the resulting optimal control $\boldsymbol{u}_{i}^{*}$ is applied without delay until $t_{i+1}$, where the process is repeated. However, for achieving optimal performance and good stability properties, problem $\mathcal{P}_{N-i}$ needs to be solved instantaneously. We refer to this hypothetical case as the ideal NMPC (INMPC). Algorithm (1) details its main steps.

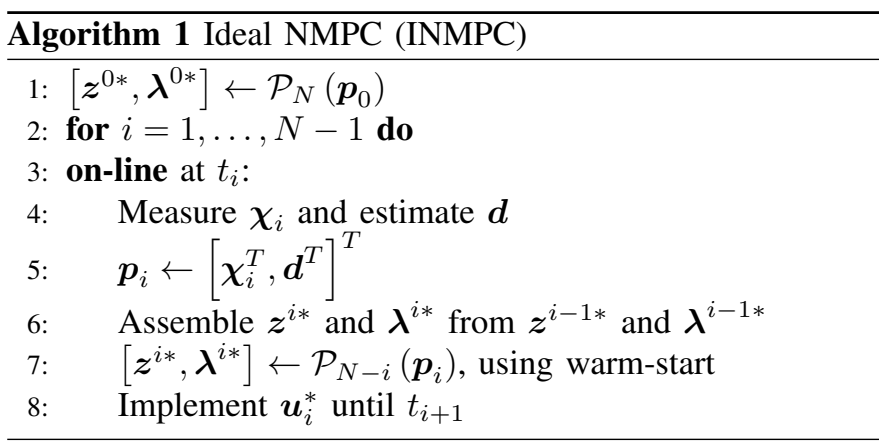

It should be noted that in practical applications $\mathcal{P}_{N-i}$ may be computationally expensive to solve. This implies that the control $\boldsymbol{u}_{i}^{*}$ cannot be applied just after $\boldsymbol{p}_{i}$ is measured, but after $\mathcal{P}_{N-i}$ is solved. The delay in calculating the new solution may lead to sub-optimum trajectories, failure to meet constraints, or in some instances instabilities of the solution [18]. This motivates the introduction of sensitivity-based methods.

2) Sensitivity-based NMPC (SbNMPC): If $\Delta \boldsymbol{p}_{i}$ is small, it is not really necessary to solve $\mathcal{P}_{N-i}$ at each time sample. In the neighborhood of the preceding optimal solution (that computed at $t_{i-1}$ ), parametric sensitivities can be calculated to rapidly update the optimal solution at $t_{i}$ using a first-order approximation. This fast trajectory update is performed by solving $\mathcal{Q}_{N-i}$ and updating the optimal solution with (10).

A virtue of this method is that $\mathcal{F}^{i *}$ can be evaluated in the background (between $t_{i-1}$ and $t_{i}$ ) at the solution of $\mathcal{P}_{N-i-1}$ assuming the estimated parameters $\boldsymbol{p}_{i}$ [10], [19]. Then, online (at $t_{i}$ ) the optimal solution is updated by solving $\mathcal{Q}_{N-i}$ almost instantaneously right after collecting $\boldsymbol{p}_{i}$ measurements.

This method is commonly referred as sensitivity-based NMPC (SbNMPC). Algorithm (2) shows the main steps of SbNMPC, where $\tau_{\text {opt }}$ and $\tau_{\text {infs }}$ are the pre-defined tolerances for the optimality and feasibility criteria, respectively. 


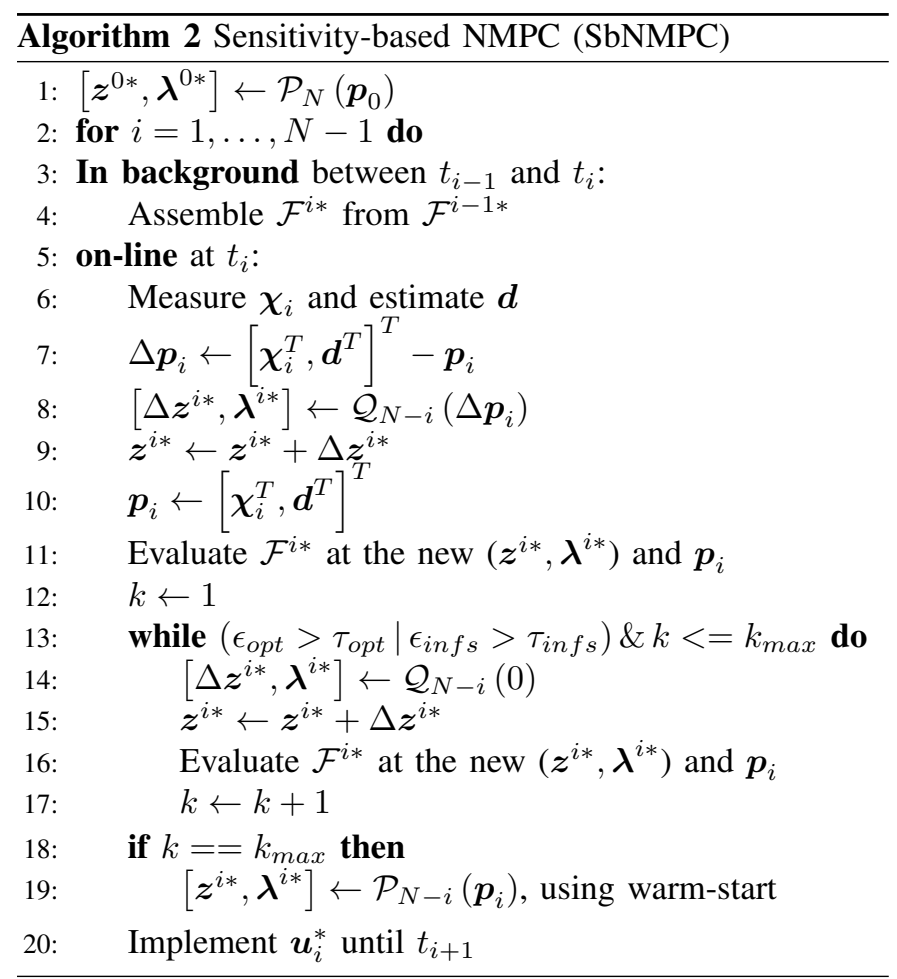

Note that the contingency steps 18 and 19 are optional. An alternative is to not update the trajectory if the optimality and feasibility criteria are not satisfied after $k_{\max }$ QP iterations.

\section{NMPC GUIDANCE STRATEGIES FOR A TIME-CONSTRAINED CDO}

In this Section the generic optimal control (1) problem is particularised for an already initiated (i.e., where the top of descent, TOD, has been overflown) CDO subject to a time constraint at a single metering fix. Then, a method to estimate the parameters of the model representing the wind is proposed.

\section{A. Optimal control problem formulation}

The state vector $\boldsymbol{x}=[t, v, h]$ is composed of time, true airspeed (TAS), and altitude; the control vector $\boldsymbol{u}=[\gamma, T, \beta]$ is composed of the aerodynamic flight path angle, engine thrust, and speed brakes deflection. The flight path angle is the control that is used by the aircraft to modulate energy (i.e., exchange potential energy for kinetic energy and vice-versa), whereas thrust and speed brakes are used to add and remove energy.

Different from typical approaches, the independent variable is the distance to go $(s)$ and not the time. This change is motivated by the fact that during an ideal CDO, with no intervention from the air traffic controllers (ATC) except for the assignment of the CTA, the aircraft will follow a "closedloop" route and the remaining distance to go will be known. In addition, this formulation eases the definition of constraints at the different waypoints (located at given $s$ ) of the route.

For the remainder of this document, the optimal control problem will be formulated in the continuous domain. However, it is straightforward to discretise it in the form of (1).
The dynamics of $\boldsymbol{x}$ are expressed by the following set of ordinary differential equations, considering a point-mass representation of the aircraft reduced to a "gamma-command" model, where vertical equilibrium is assumed (lift balances weight) and the cross and vertical winds are neglected:

$$
\boldsymbol{f}=\frac{\mathrm{d} \boldsymbol{x}}{\mathrm{d} s}=\frac{\mathrm{d} \boldsymbol{x}}{\mathrm{d} t} \frac{\mathrm{d} t}{\mathrm{~d} s}=\left[\begin{array}{c}
1 \\
\frac{T-D}{m}-g \sin \gamma \\
v \sin \gamma
\end{array}\right] \frac{1}{v \cos \gamma+w}
$$

where $D: \mathbb{R}^{n_{x} \times n_{u}} \rightarrow \mathbb{R}$ is the aerodynamic drag; $g$ is the gravity acceleration and $m$ the mass, of which is assumed to be constant because the fuel consumption during a descent is a small fraction of the total $m$ [20]. The longitudinal component of the wind $w: \mathbb{R} \rightarrow \mathbb{R}$ is modelled by a spline [21]:

$$
w(h)=\sum_{i=1}^{n_{c}} c_{i} B_{i}(h)
$$

$B_{i}, i=1, \ldots, n_{c}$, are the B-spline basis functions and $\boldsymbol{c}=\left[c_{1}, \ldots, c_{n_{c}}\right]$ are control points of the smoothing spline. It should be noted that the longitudinal wind has been modelled as a function of the altitude only, as done in similar works [22].

Since the total flight time is fixed by the CTA, the goal is to minimise a weighted sum of the fuel consumption and speed brakes use (which leads to airframe noise and pilot workload) for the remaining distance to go. Therefore, the stage cost is:

$$
\ell=\frac{f_{f}+K_{\beta} \beta}{(v \cos \gamma+w)}
$$

where $f_{f}: \mathbb{R}^{n_{x} \times n_{u}} \rightarrow \mathbb{R}$ is the fuel flow and $K_{\beta}$ a parameter that determines how much the use of speed brakes is penalised.

In the formulation considered herein, the generic path constraints ensure that the aircraft airspeed remains within operational limits, and that the maximum and minimum descent gradients, thrust and speed brakes are not exceeded:

$$
\boldsymbol{h}=\left[\begin{array}{c}
v_{C A S}-v_{C A S_{\min }} \\
\mathrm{VMO}-v_{C A S} \\
\mathrm{MMO}-M \\
0-\gamma \\
\gamma-\gamma_{\min } \\
T-T_{\min } \\
T_{\max }-T \\
\beta \\
1-\beta
\end{array}\right] \geq\left[\begin{array}{l}
0 \\
0 \\
0 \\
0 \\
0 \\
0 \\
0 \\
0 \\
0
\end{array}\right]
$$

where $v_{C A S}: \mathbb{R}^{n_{x}} \rightarrow \mathbb{R}$ is the calibrated airspeed (CAS) and $M: \mathbb{R}^{n_{x}} \rightarrow \mathbb{R}$ is the Mach number, both functions of the state vector; $v_{C A S_{m i n}}$ and VMO are the minimum and maximum operative CAS, respectively; MMO is maximum operative Mach; $\gamma_{\min }$ is the minimum descent gradient; $T_{\min }$ : $\mathbb{R}^{n_{x}} \rightarrow \mathbb{R}$ and $T_{\max }: \mathbb{R}^{n_{x}} \rightarrow \mathbb{R}$ are the idle and maximum thrust, respectively; $\beta=0$ and $\beta=1$ indicate that speed brakes are retracted and fully extended, respectively. 
Different alternatives can be used to model the aircraft performance functions $T_{\min }, T_{\max }, D$ and $f_{f}$ and their respective parameters. In this paper, the EUROCONTROL's base of aircraft data (BADA) v4 model has been adopted [23]. However, BADA v4 does not include a model for the effects of the speed brakes on the drag coefficient $C_{D}$. As a workaround, in this paper the contribution of the speed brakes is modelled as an extra linear term $C_{D_{\beta}} \beta$ in the generic BADA v4 drag coefficient model, where $C_{D_{\beta}}$ is a coefficient representing the increase in drag coefficient for unit of speed brakes deflection.

Terminal constraints fix the state vector at the metering fix:

$$
\boldsymbol{\psi}=\left[\begin{array}{c}
t-\mathrm{CTA} \\
v_{C A S}-v_{C A S_{f}} \\
h-h_{f}
\end{array}\right]=\left[\begin{array}{l}
0 \\
0 \\
0
\end{array}\right]
$$

$v_{C A S_{f}}$ and $h_{f}$ are the CAS and altitude at the metering fix.

The vector of model parameters includes the control points of the spline approximating the longitudinal wind and CTA, i.e., $\boldsymbol{d}=\left[\boldsymbol{c}^{T}, \mathrm{CTA}\right]^{T}$. This definition allows the optimal trajectory to be updated whenever an improved wind forecast is available or the CTA is modified by ATC.

The descent is divided into several phases, defined between two consecutive waypoints of the lateral route with associated speed and/or altitude constraints. In each phase, different operational constraints may apply and may be modelled in the form of additional path and terminal constraints, which are appended to (15) and (16), respectively. Finally, in the presence of more than one phase, link constraints are added to ensure continuity of the state vector across phases (i.e., the state vector at the first point of one phase must be identical to the state vector at the last point of the preceding one).

\section{B. On-line wind profile estimation}

By definition of $\mathcal{P}_{N-i}$ and $\mathcal{Q}_{N-i}$, the optimal trajectory is an implicit function of the parameters vector. In the model proposed in this paper, the parameters vector includes the control points of a spline that fits an approximated longitudinal wind profile. Accordingly, whenever an improved wind forecast is available, the trajectory can be updated either by solving a rigorous NLP problem or by using parametric sensitivities.

Given $n_{o}$ wind observations at different altitudes $\left(\hat{h}_{k}, \hat{w}_{k}\right)$, $k=1, \ldots, n_{o}$, and a vector of fixed knots, the optimal location of the control points $c_{k}, k=1, \ldots, n_{c}$, that minimise the curvature of the spline while bounding the approximation error is obtained by solving a weighted least-squares fitting problem:

$$
\begin{array}{ll}
\min _{c_{k}, k=1, \ldots, n_{c}} & \int_{\text {s.t }} w^{\prime \prime}(h)^{2} \mathrm{~d} h \\
& \sum_{k=1}^{n_{o}} \omega_{k}\left(w\left(\hat{h}_{k}\right)-\hat{w}_{k}\right)^{2} \leq \varepsilon
\end{array}
$$

where $\varepsilon$ specifies the trade-off between smoothness and accuracy of the approximation. The weights associated with the observations can be defined in many different ways. In this paper, the weights are updated at each time sample $t_{i}$ according to $\omega_{k}=\Lambda^{t_{k}-t_{i}}$, where $t_{k}$ is the time sample when the observation $\left(\hat{h}_{k}, \hat{w}_{k}\right)$ was obtained. The forgetting factor $\Lambda \in[0,1]$ weights the more recent measurements so that old observations are discounted at an exponential rate.

Before starting the descent, the initial spline approximating the longitudinal wind profile is generated by solving (17) with data from a numerical weather forecast. During the course of the descent, the wind at the current altitude is measured and added to the set of observations. Then, weights are updated and problem (17) is solved with negligible time. This results in a new $c$, which allows the optimal trajectory to be updated according to a better estimation of the actual wind conditions.

\section{EXPERIMENT SETUP}

Several descents subject to CTA for a realistic vertical profile, which includes several altitude and speed constraints, were simulated to asses the performance of INMPC and $\mathrm{SbNMPC}$ in the presence of errors in the wind forecast.

\section{A. Scenario}

The Denver (DEN) international airport (Colorado) was selected as the scenario for the experiment. The lateral route and the vertical profile were inspired by the BOSSS TWO standard arrival procedure at DEN (see Fig. 1).

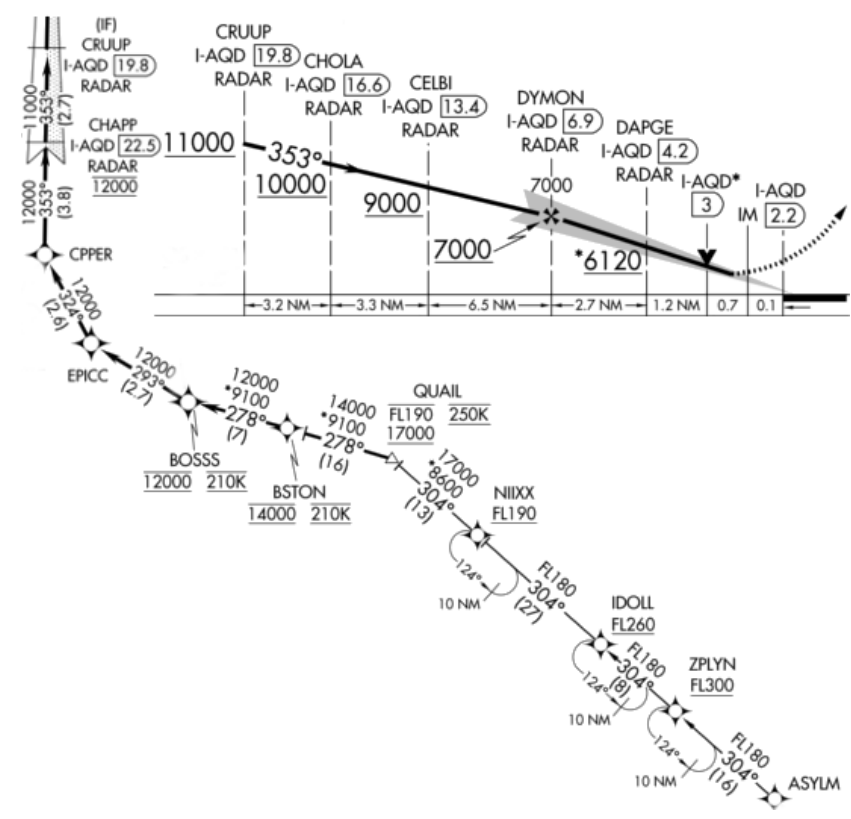

Fig. 1. BOSSS TWO standard arrival procedure (source: US AIP)

In this experiment, the metering fix where CTAs were assigned by an hypothetical ATC during the simulations was the final approach point (DYMON). Terminal constraints (16) for the optimal control problem were set at DYMON, with $h_{f}=7000 \mathrm{ft}, v_{C A S_{f}}=200 \mathrm{kt}$ and the time fixed to the CTA.

From all the waypoints of the route, only the altitude and speed constraints at QUAIL, BOSSS, CHAPP and DYMON were modelled. Table I wraps up the phases required to represent this specific vertical profile and their associated 
TABLE I

FLIGHT PHASES FOR THE BOSSS TWO PROCEDURE MODEL

\begin{tabular}{|c|c|c|c|c|}
\hline Phase & Start $s$ & End $s$ & Path constraints & Terminal constraints \\
\hline 01 & $s_{\mathrm{TOD}}$ & QUAIL & $v_{C A S}-250 \mathrm{kt} \geq 0$ & $\begin{array}{c}v_{C A S}-250 \mathrm{kt}=0 \\
h-17000 \mathrm{ft} \geq 0 \\
\text { FL190-h } \geq 0\end{array}$ \\
\hline 02 & QUAIL & BOSSS & $\begin{array}{l}250 \mathrm{kt}-v_{C A S} \geq 0 \\
v_{C A S}-210 \mathrm{kt} \geq 0\end{array}$ & $\begin{array}{c}v_{C A S}-210 \mathrm{kt}=0 \\
h-12000 \mathrm{ft}=0\end{array}$ \\
\hline 03 & BOSSS & CHAPP & $\begin{array}{l}210 \mathrm{kt}-v_{C A S} \geq 0 \\
v_{C A S}-200 \mathrm{kt} \geq 0\end{array}$ & $h-12000 \mathrm{ft}=0$ \\
\hline 04 & CHAPP & DYMON & $\begin{array}{l}210 \mathrm{kt}-v_{C A S} \geq 0 \\
v_{C A S}-200 \mathrm{kt} \geq 0\end{array}$ & Eq. (16) \\
\hline
\end{tabular}

path and terminal constraints, which were considered during the trajectory optimisation process in addition to the generic constraints of the CDO model presented in SectionIII-A.

For the generic path constraints (15), the minimum descent gradient and calibrated airspeed were set to $-7^{\circ}$ and $200 \mathrm{kt}$, respectively. In addition, the values for VMO and MMO were obtained from the BADA v4 global parameters file.

\section{B. Case studies}

Accurate wind data can be obtained from the rapid refresh (RAP) forecast/analysis system of the National Oceanic and Atmospheric Administration (NOAA). This system generates numerical weather forecasts hourly for look-ahead times up to +18 hours in a $13 \mathrm{~km}$ resolution grid covering North America and for 50 vertical levels extending up to $10 \mathrm{hPa}$. Sightly different, RAP analyses, which reproduce the actual weather conditions, are generated hourly by using observations gathered from commercial aircraft, balloons, radars and satellites.

Historical RAP wind forecasts for look-ahead times of +1 , +3 and +6 hours during one year (from May 2017 to May 2018) were compared with actual wind data as reported by the corresponding RAP analysis. From these data, the root-meansquare error (RMSE) of each wind forecast over the region of interests (Denver) up to FL400 was computed.

Then, for each one of the look-ahead times considered in this paper, the corresponding set of RAP forecasts was ranked according to RMSE, and 5 representative case studies were selected: the forecasts with maximum and minimum RMSE, and the forecasts associated to quartiles Q1, Q2 and Q3. Table II lists the 15 case studies of the experiment.

\section{Generic simulation workflow}

The experiment simulated an Airbus A320-214 cruising at FL360 and Mach 0.78. Well before starting the descent, the FMS computed the optimal descent trajectory to DYMON for a typical cost index of $30 \mathrm{~kg} \mathrm{~min}^{-1}$ [24], discretising the continuous optimal control problem into $N=60$ time samples. The initial plan was computed considering a smoothing spline for the longitudinal wind profile $^{1}$ that approximated the RAP

\footnotetext{
${ }^{1}$ It should be noted that the longitudinal wind profile was computed assuming a constant track of $304^{\circ}$ all along the route for the sake of simplicity.
}

TABLE II

CAse studies

\begin{tabular}{|c|c|c|c|c|}
\hline Case Study & Look-ahead time & Forecast generation & Metric & RMSE [kt] \\
\hline 00 & \multirow{5}{*}{+1} & 17-09-26 06:00 & $\min$ & 0.2 \\
\hline 01 & & 17-07-02 12:00 & Q1 & 1.4 \\
\hline 02 & & 17-10-05 18:00 & Q2 & 1.9 \\
\hline 03 & & 18-03-14 12:00 & Q3 & 2.7 \\
\hline 04 & & 18-04-21 00:00 & $\max$ & 7.4 \\
\hline 05 & \multirow{5}{*}{+3} & 17-08-27 18:00 & $\min$ & 1.0 \\
\hline 06 & & $18-01-18 \quad 06: 00$ & Q1 & 2.5 \\
\hline 07 & & 18-02-13 12:00 & Q2 & 3.3 \\
\hline 08 & & 18-02-01 18:00 & Q3 & 4.3 \\
\hline 09 & & 18-04-21 00:00 & $\max$ & 14.5 \\
\hline 10 & \multirow{5}{*}{+6} & 17-07-04 12:00 & $\min$ & 1.4 \\
\hline 11 & & 17-10-13 06:00 & Q1 & 3.3 \\
\hline 12 & & 17-09-27 18:00 & Q2 & 4.1 \\
\hline 13 & & $17-12-26 \quad 18: 00$ & Q3 & 5.2 \\
\hline 14 & & 17-09-19 18:00 & $\max$ & 14.2 \\
\hline
\end{tabular}

wind forecast data. As a result of this optimisation process, the best distance to go from the top of descent, $s_{\mathrm{TOD}}$, and the optimal time of arrival at DYMON were obtained. In addition, the energy-neutral time window ${ }^{2}$ from $s_{\mathrm{TOD}}$ to DYMON was also computed and sent to the hypothetical ATC.

For this simulation, it was assumed that the ATC assigned a CTA at DYMON when the aircraft reached the planned TOD. The CTA was generated as a random value from a normal distribution that fitted within the energy-neutral time window. Subsequently, the FMS set the CTA as a terminal constraint for the time variable in Eq. (16) and calculated the optimal descent trajectory from the current state to DYMON by solving $\mathcal{P}_{N}$.

All simulations started with the aircraft located at the TOD, ready to start the execution of the optimal descent trajectory using either of the NMPC strategies. During the execution, the aircraft encountered the actual wind profile, which was different from the initial forecast used by the FMS. As a result, the aircraft experienced state perturbations from the initial plan when applying the optimal control. These state perturbations were corrected by updating the trajectory at each time sample. In addition, these updates also took into account changes in the control points of the smoothing spline representing the wind profile. The control points were recalculated by solving (17) at each time sample, as fresh actual wind observations were notionally gathered by the sensors of the aircraft.

\section{REsults}

Section V-A shows examples of trajectory updates after perturbing the parameters vector. Section V-B thoroughly describes an illustrative example of the simulation. Finally, the aggregated results are discussed in Section V-C.

\footnotetext{
${ }^{2}$ The energy-neutral time window from a state to a metering fix is defined as the difference between the latest and earliest time of arrival that could be achieved without requiring neither additional thrust nor speed brakes use throughout the descent. Previous works show that energy-neutral time windows up to 4 minutes can be obtained for certain flight conditions [25].
} 


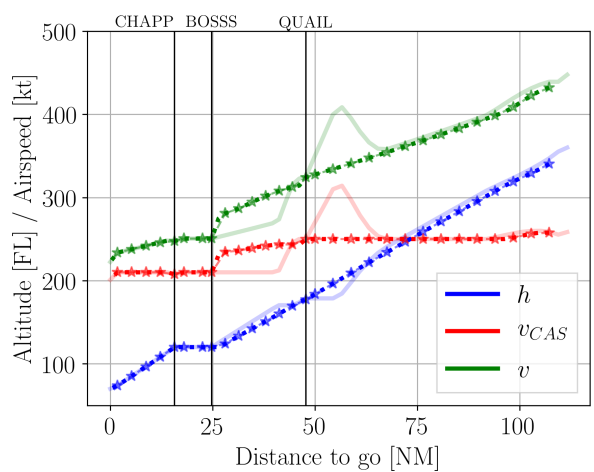

(a) $\delta \boldsymbol{\chi}_{1}=[0,0,-1000 \mathrm{ft}]$

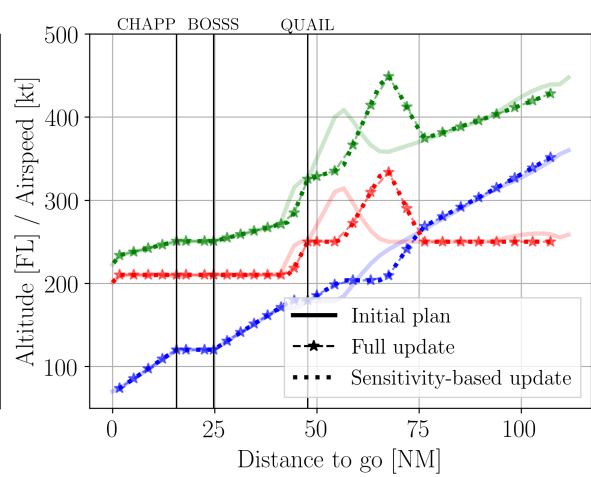

(b) $\delta c$ (actual minus forecast of Fig. 4)

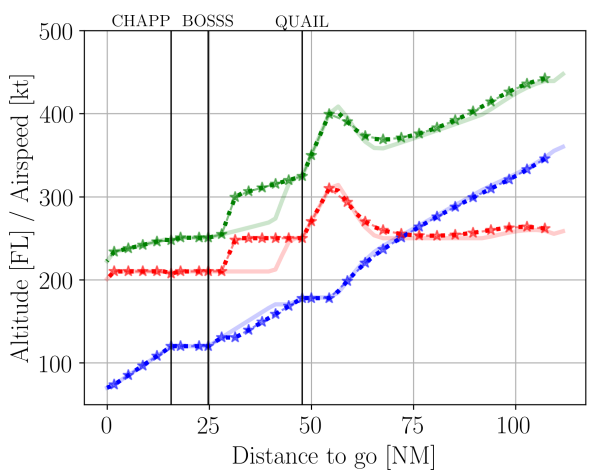

(c) $\delta \mathrm{CTA}=-30 \mathrm{~s}$

Fig. 2. Optimal trajectory updates for perturbations in the parameters vector (case study 04)

\section{A. Examples of trajectory update}

The NMPC strategies presented in Section II-C update the optimal trajectory at each time sample for perturbations in the vector of NLP parameters $\boldsymbol{p}$. By definition, any perturbation on an element of $\boldsymbol{p}$ would result in a new optimal trajectory.

Remember that for the model proposed in Section III-A, the vector of NLP parameters at time sample $t_{i}$ is composed by the current state of the aircraft $\chi_{i}$ and the parameters of the model $\boldsymbol{d}$. In turn, $\boldsymbol{d}$ includes the control points of a spline approximating the longitudinal wind profile $c$ and the CTA.

Fig. 2 shows the initial (unperturbed) trajectory for the case study 04 (the largest RMSE of a +1 hour RAP wind forecast), and compares the trajectory of a full update and a sensitivitybased update for perturbations in the different elements of $\boldsymbol{p}$. Fig. 4 shows the initial wind profile forecast and the actual wind profile for this particular case study. According to this figure, during the first part of the descent (from FL360 to FL200) the aircraft will encounter a tail wind significantly stronger than that reported by the initial wind forecast.

To study the impact of perturbations in the parameters vector, a deviation in the state vector, wind, or CTA was inserted at the TOD: The altitude deviation was $1000 \mathrm{ft}$ below the unperturbed trajectory; wind deviation was the difference between the control points of the splines approximating the RAP forecast and analysis data; and the CTA deviation was ATC requiring the aircraft to arrive 30 seconds earlier than initially requested. The remainder of this section describes, and Fig. 2 illustrates, the trajectory update for each perturbation.

Fig. 2 shows that the optimal trajectories resulting from sensitivity-updates are almost identical to those obtained from a full update, even for large perturbations in elements of $\boldsymbol{p}$.

According to Fig. 2(a), when the actual altitude of the aircraft was $1000 \mathrm{ft}$ below the initial plan (lacking of energy), the energy loss rate needed to be reduced in order to satisfy the altitude and speed constraints enforced at QUAIL (see Table I). The most fuel efficient method to accomplish that consists of reducing the airspeed with the elevator and consequently the aerodynamic drag, which is the main cause of energy loss.

However, this sustained reduction in airspeed delayed the aircraft with respect to the CTA. The induced time error was compensated afterwards by flying at a higher speed from QUAIL to BOSS, yet releasing the same amount of energy than initially planned by following a different energy profile.

Fig. 2(b) shows trajectory updates for perturbations in the control points of the spline approximating the wind profile. The updated trajectory avoided the altitude interval from FL200 to FL260 as much as possible, where the tail wind was significantly stronger than forecast. In order to accomplish that, potential energy (altitude) was exchanged for kinetic energy (airspeed) at the maximum descent gradient by means of elevator control. This process was only applied down to FL200, since the extra kinetic energy would bring the aircraft to DYMON earlier than the CTA. This kinetic energy was rapidly released by means of a level-off at idle thrust at FL200.

Finally, according to Fig. 2(c) the optimal way to arrive 30 seconds earlier than initially planned consisted of flying at the maximum allowed airspeed from QUAIL to BOSSS (250 kt), and then releasing the excess of kinetic energy by executing a level-off at idle thrust just before BOSSS, in order to satisfy its associated hard altitude and speed constraints (see Table I).

It should be noted that for the three examples presented in Fig. 2, the trajectories resulting from a NMPC trajectory update reached DYMON at the enforced time and energy using only energy modulation by means of elevator control.

\section{B. Example of simulation}

Using the same case study as the previous section, this section will describe the behavior of the guidance strategies.

Fig. 3 shows the initially planned trajectory (computed at the TOD) and the executed trajectory, for the two NMPC variants proposed in this paper and for the open-loop strategy (OL). The OL strategy simply applies the optimal control of the initial plan throughout the descent, without neither monitoring state deviations nor updating the optimal descent trajectory.

The light solid lines in all three panels of Fig. 2 and in Fig. 3 are identical to each other, i.e., the initially planned trajectory. Then, the slightly darker solid lines in Fig. 3 represent the plans resulting from trajectory updates at the time samples 10 and 40. The plans generated at the remaining 57 time samples are not shown for the sake of clarity, even if being 


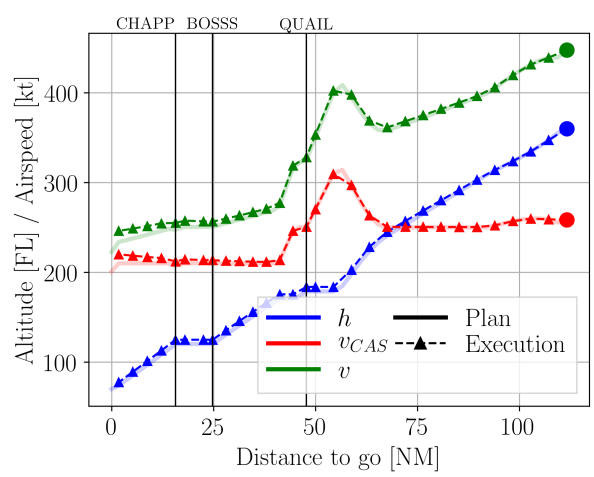

(a) Open-loop

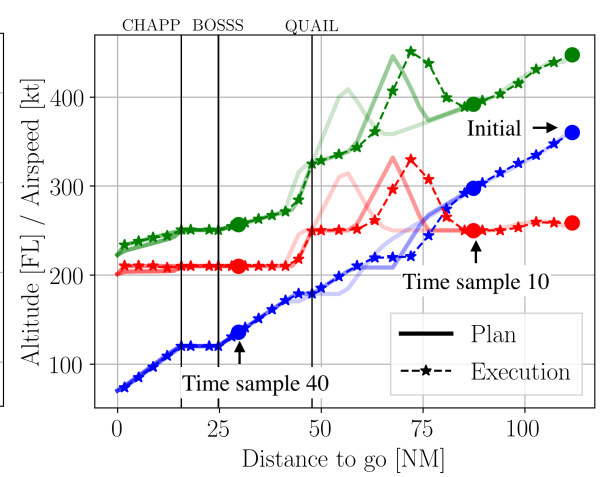

(b) INMPC

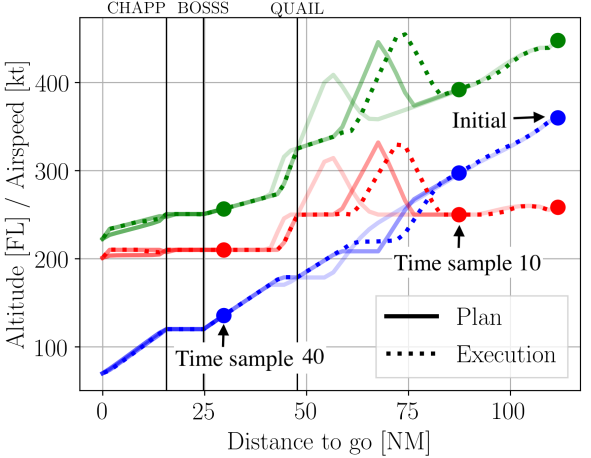

(c) SbNMPC

Fig. 3. Planned and executed trajectories by control method (case study 04)

computed. As observed in Fig. 2, there is little difference in the trajectories generated by using full and sensitivity-based updates. Accordingly, the dashed lines in Fig. 3 (the execution using either INMPC or SbNMPC) will be almost identical.

Before updating the trajectory at each time sample, the NMPC guidance system recalculates the control points of the spline that approximates the wind profile, based on the actual wind sensed at the current altitude. The wind profile used by the NMPC trajectory optimiser converges to the actual wind profile as wind measurements are collected during the descent. This can be observed in Fig. 4, which shows the initial wind profile (fitting the RAP forecast data), the wind profile at the time samples 10 and 40, and the actual wind profile encountered by the aircraft during the course of the descent.

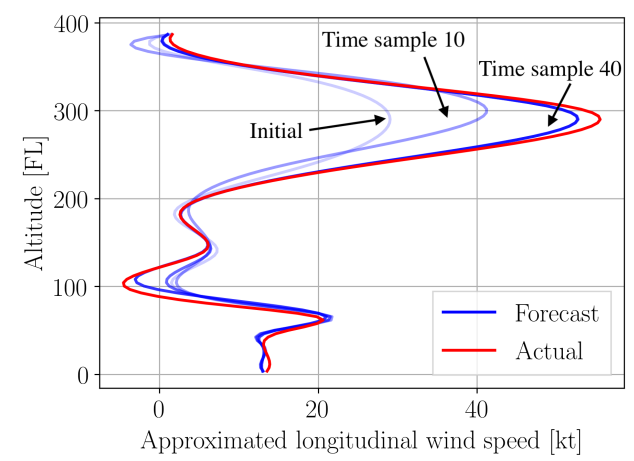

Fig. 4. RAP wind forecast and analysis for the case study 04)

In both INMPC and SbNMPC, the plan resulting from the trajectory update performed at time sample 10 aimed to start an energy modulation process around FL260 to avoid the altitude interval with extreme and unexpected tail wind, given the best available wind profile estimation at that time sample (see Fig. 4). However, according to the executed trajectory, a later trajectory update (not shown in Fig. 3) requested to start exchanging potential for kinetic energy around $1000 \mathrm{ft}$ earlier. After QUAIL, the updated trajectory demanded a sightly lower speed than that of the initial plan, in order to compensate the unexpected head wind in the altitude interval FL170-7000 ft.
It should be noted that the optimal control applied at each time sample is based on the best estimation of the wind profile at the current altitude. However, the actual wind conditions that the aircraft will encounter at lower altitudes are still unknown. Even if the control applied at each time sample is optimal for the estimated wind, it is sub-optimal for the actual (unknown) wind conditions. This can be observed by comparing Figs. 3(b) and 2(b): the executed trajectory (resulting from applying the optimal control for the best estimated wind at each time sample), and the trajectory update computed at the TOD with information of the actual wind, are similar yet not identical.

For this particular case study, the open-loop trajectory arrived at the metering 27 seconds earlier than the CTA and $600 \mathrm{ft}$ above the enforced specific energy level. For the INMPC and SbNMPC, the aircraft arrived at DYMON with negligible time and energy error using minimum thrust and speed brakes.

\section{Aggregated results}

This section presents the performance metrics for the case studies of the experiment. Fig. 5(a) shows the time error at the metering fix (DYMON) with respect to the enforced CTA for the INMPC and SbNMPC strategies. The time error that would be achieved by applying the optimal control resulting from the initial plan in open-loop is also shown. Analogously, Fig. 5(b) shows the specific energy $\left(E_{s}\right)^{3}$ error at DYMON.

According to Fig. 5(a), and as expected, for each look-ahead forecast time the case studies with maximum RMSE lead to the largest time errors when applying the control of the initial plan in open-loop. In addition, the larger the look-ahead time, the more time error is observed. In fact, the largest time error was realised for the case study with maximum RMSE of the +6 look-ahead time set (case study 14). For this particular case study, the time error for the OL strategy was around $80 \mathrm{~s}$.

When using strategies based on NMPC, the time error was drastically reduced. For all case studies analysed herein the time error was lower than $5 \mathrm{~s}$ when using these strategies except for the case study 14, in which the time error was 20 $\mathrm{s}$ (still four times lower than for the open-loop strategy).

\footnotetext{
${ }^{3}$ The specific energy is defined as the total energy of the aircraft divided by the aircraft weight. By definition, the units of the specific energy are $\mathrm{ft}$.
} 

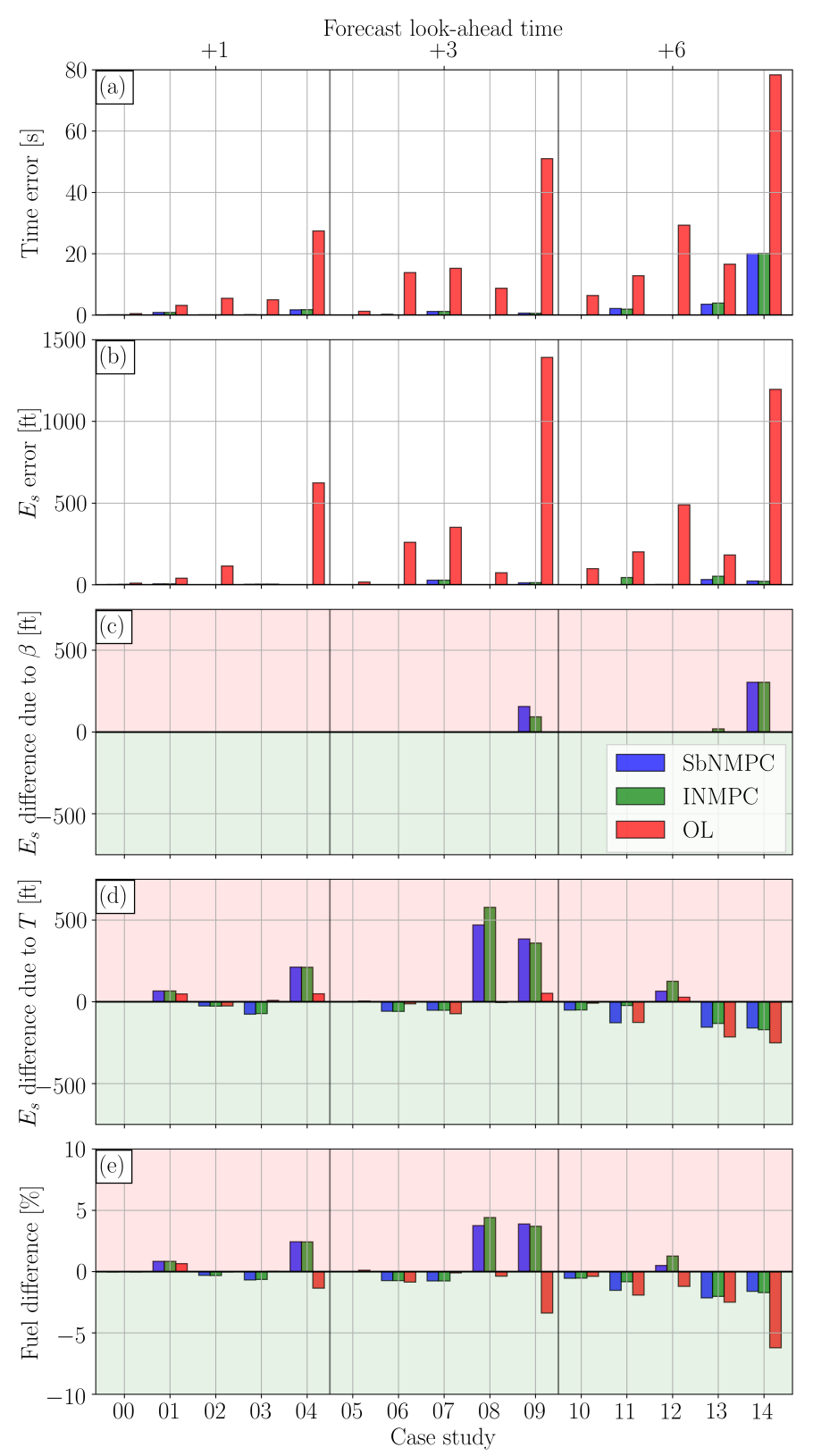

Fig. 5. Aggregated results at metering fix by case study

For this particular case study, the aircraft encountered a strong tail wind at low altitude, which was not predicted by the weather forecast. Since the active optimal trajectory between BOSSS and DYMON was at the minimum allowed speed of $200 \mathrm{kt}$, it was not possible to further decelerate and the time error could not be nullified. If the guidance strategy had known in advance the actual wind conditions from observations transmitted by preceding aircraft at low altitudes, then the trajectory recalculation would have commanded an earlier deceleration to compensate for the tail wind. In this experiment, the wind profile used by the FMS was updated only with ownship wind measurements. Consequently, the actual wind conditions downstream were not known a priori.
Similar results were observed for the specific energy error. According to Fig. 5(b), the metering fix could be achieved with specific energy errors up to $1400 \mathrm{ft}$ by implementing the control of the initial plan in open-loop. Conversely, guidance strategies based on NMPC achieved the metering fix with negligible specific energy error, typically lower than $20 \mathrm{ft}$.

Figs. 5(a) and 5(b) also show that the performance of the SbNMPC in terms of energy and time errors is equivalent to that of the INMPC. Results agree with those shown in previous sections, where the trajectory updates based on sensitivities were similar to those obtained by solving the NLP problem.

In the model proposed in this paper, the optimal control computed at each $t_{i}$ could claim to modulate energy with the elevator or to add/remove energy to/from the system by means of additional thrust or speed brakes. Ideally, the NMPC optimiser will attempt to obtain an energy-neutral trajectory.

In certain conditions, however, energy modulation by means of elevator control may not be sufficient to obtain a solution satisfying all the constraints. In this case, the NMPC trajectory optimiser would calculate the optimal amount of energy to be added or removed in terms of fuel consumption and speed brakes use such that all constraints are satisfied.

Fig. 5(c) shows, for each case study, the difference between the executed trajectory and the initial plan, in terms of total specific energy removed by deployment of the speed brakes. Positive values indicate that trajectory updates removed more energy by means of speed brakes than initially planned. Fig. 5(d) shows the difference in specific energy added by thrust between the initial plan and executed plan, with positive values indicating more thrust was used than initially planned.

When the aircraft deviates from the active plan, the thrust idle for the actual flight conditions may be different from that of the initial plan at the same $t_{i}$. Therefore, the thrust applied at $t_{i}$ according to the plan could be sightly different from the actual idle thrust. State deviations are the cause of the specific energy differences shown in Fig. 5(d) for the OL strategy.

According to Figs. 5(c) and 5(d), for the case studies with large RMSE in the wind forecast (04, 09 and 14), thrust or speed brakes were required. For most of the remaining case studies, energy modulation was sufficient. Yet, the amounts of energy added/removed by the NMPC strategies are optimal in terms of fuel consumption and use of speed brakes.

Case study 09 is the only simulation where both additional thrust and use of speed brakes were required. During the first part of the descent, a trajectory update generated with the best available estimate of the wind profile at that time sample required the use of speed brakes to remove energy. Then, at lower altitudes, with a different sensed wind, a new trajectory update required thrust to increase the energy of the aircraft. A better wind forecast, for example by incorporating the broadcasts of the sensed wind by preceding aircraft on the arrival procedure, should somewhat mitigate this issue.

Fig. 5(e) shows the difference in fuel consumption between the executed trajectory and the initial plan, with negative values indicating fuel savings and positive values indicating that additional fuel was required. Results shown in Fig. 5(e) 
agree with those of Fig. 5(d): the cases studies that required additional specific energy (i.e., thrust), resulted in extra fuel consumption. Interestingly, compared to the open-loop execution, the extra fuel required by the NMPC guidance strategies to compensate for up to 80 seconds or $1400 \mathrm{ft}$ of error is less than $5 \%$ of the total fuel consumed during the descent.

\section{CONCLUSIONS}

This paper shows the application of a non-linear model predictive control (NMPC) guidance strategy that uses parametric sensitivities to update the optimal trajectory during the descent of an aircraft subject to a time constraint at a metering fix.

Results show that trajectory updates resulting from solving a non-linear programming (NLP) problem are very similar to those obtained by using parametric sensitivities. Yet, the later optimisation method is simpler, faster and more robust.

In addition, results show that for small errors in the forecast of the wind profile, energy modulation through elevator control typically suffices to compensate induced time and energy errors. However, for large errors in the wind forecast, some energy needs to be added or removed with throttle or speed brakes. In this case, the NMPC strategy proposed in this paper computes the optimal energy to be added or removed such that fuel consumption and use of speed brakes is minimised.

Promising results from simulations show that energy and time errors up to $1400 \mathrm{ft}$ and 80 seconds, respectively, could be compensated for by less than a $5 \%$ increase in the fuel consumed during the arrival operation.

In future work, the performance of these NMPC guidance strategies will be assessed for a large set of case studies, in order to obtain statistically meaningful results. Future work may also assess the effects of wind networking (i.e., taking advantage of wind observations gathered by nearby aircraft to improve the on-board wind profile estimations) on the performance metrics. Finally, other variants of the proposed model including coefficients of the aircraft performance model in the vector of NLP parameters could be also investigated.

\section{ACKNOWLEDGMENT}

The authors acknowledge the technical support during the development of the guidance algorithms presented in this paper of PhD. Joel Andersson, one of the main authors of the CasADi software package. Much appreciation goes out to Prof.Dr.-Ing. Wolfgang Marquardt and PhD. Ralf HannemannTamás for their expertise in the NMPC field and advice.

\section{REFERENCES}

[1] A. E. Bryson and Y.-C. Ho, Applied optimal control : optimization, estimation, and control. New York, USA: Taylor and Francis Group, 1975.

[2] R. Dalmau, X. Prats, R. Verhoeven, F. Bussink, and B. Heesbeen, "Performance comparison of guidance strategies to accomplish RTAs during a CDO," in 36th IEEE/AIAA Digital Avionics Systems Conference (DASC), St. Petesburg, FL, 2017.

[3] P. M. A. de Jong, N. de Gelder, R. Verhoeven, F. J. L. Bussink, R. Kohrs, M. M. van Paassen, and M. Mulder, "Time and Energy Management During Descent and Approach: Batch Simulation Study," Journal of Aircraft, vol. 52, no. 1, pp. 1-14, 2014.
[4] M. Diehl, H. J. Ferreau, and N. Haverbeke, "Efficient Numerical Methods for Nonlinear MPC and Moving Horizon Estimation Problem Formulation," in Workshop on Assessment and Future Directions of NMPC, Pavia, Italy, 2008, pp. 391-417.

[5] L. A. Weitz and X. Bai, "Using Model Predictive Control for Trajectory Optimization and to Meet Spacing Objectives," in AIAA Guidance, Navigation, and Control Conference, Kissimmee, FL, 2018.

[6] E. Suwartadi, V. Kungurtsev, and J. Jäschke, "Sensitivity-Based Economic NMPC with a Path-Following Approach," Processes, vol. 5, no. 1, p. 8, 2017.

[7] T. J. Kim and D. G. Hull, "Optimal control design that accounts for model mismatch errors," in AAS/MAA Space Flight Mechanics Meeting, Albuquerque, New Mexico, 1995.

[8] T. A. Wetzel, "Development of a finite difference neighboring optimal control law and application to the optimal landing of a reusable launch vehicle," PhD Thesis, Iowa State University, 1996.

[9] H. J. Pesch, "Numerical computation of neighboring optimum feedback control schemes in real-time," Applied Mathematics \& Optimization, vol. 5, no. 1, pp. 231-252, 1979.

[10] L. Würth, R. Hannemann, and W. Marquardt, "An Efficient Strategy for Real-Time Dynamic Optimization based on Parametric Sensitivities," IFAC Proceedings Volumes, vol. 41, no. 2, pp. 1928-1933, 2008.

[11] — - "Neighboring-extremal updates for nonlinear model-predictive control and dynamic real-time optimization," Journal of Process Control, vol. 19, no. 8, pp. 1277-1288, 2009.

[12] I. J. Wolf and W. Marquardt, "Fast NMPC schemes for regulatory and economic NMPC A review," Journal of Process Control, vol. 44, pp. 162-183, 2016.

[13] J. T. Betts, Practical Methods for Optimal Control and Estimation Using Nonlinear Programming, 2nd ed. SIAM, 2010.

[14] H. W. Kuhn and A. W. Tucker, "Nonlinear programming," in Proceedings of the Second Berkeley Symposium on Mathematical Statistics and Probability, 1950. Berkeley and Los Angeles: University of California Press, 1951, pp. 481-492.

[15] J. Andersson, J. Akesson, and M. Diehl, "Dynamic optimization with CasADi," Proceedings of the IEEE Conference on Decision and Control, pp. 681-686, 2012.

[16] P. E. Gill, W. Murray, and M. A. Saunders, "SNOPT: An SQP algorithm for large-scale constrained optimization," Society for Industrial and Applied Mathematics (SIAM) Review, vol. 47, pp. 99-131, 2005.

[17] J. V. Kadam and W. Marquardt, "Sensitivity-Based Solution Updates in Closed-Loop Dynamic Optimization," IFAC Proceedings Volumes, vol. 37, no. 9, pp. 947-952, 2004.

[18] J. Jäschke, X. Yang, and L. T. Biegler, "Fast economic model predictive control based on NLP-sensitivities," Journal of Process Control, vol. 24, no. 8, pp. 1260-1272, 2014.

[19] S. Gros, B. Srinivasan, B. Chachuat, and D. Bonvin, "Neighbouringextremal control for singular dynamic optimisation problems. Part I: Single-input systems," International Journal of Control, vol. 82, no. 6, pp. 1099-1112, 2009.

[20] J. P. B. Clarke, N. T. Ho, L. Ren, J. A. Brown, K. R. Elmer, K. F. Zou, C. Hunting, D. L. McGregor, B. N. Shivashankara, K. Tong, A. W. Warren, and J. K. Wat, "Continuous descent approach: Design and flight test for Louisville international airport," Journal of Aircraft, vol. 41, no. 5, pp. 1054-1066, 2004.

[21] C. de Boor, "On calculating with B-splines," Journal of Approximation Theory, vol. 6, no. 1, pp. 50-62, 1972.

[22] P. M. A. de Jong, J. J. van der Laan, A. C. Veld, M. M. van Paassen, and M. Mulder, "Wind-Profile Estimation Using Airborne Sensors," Journal of Aircraft, vol. 51, no. 6, pp. 1852-1863, 2014.

[23] D. Poles, A. Nuic, and V. Mouillet, "Advanced aircraft performance modelling for ATM: Analysis of BADA model capabilities," in 29th Digital Avionics Systems Conference. Brétigny-sur-Orge (France): EUROCONTROL, 2010

[24] Airbus, "Getting to grips with the cost index," Airbus Flight Operations Support \& Line Assistance, Blagnac, France, Tech. Rep., May 1998.

[25] R. Dalmau and X. Prats, "Controlled time of arrival windows for already initiated energy-neutral continuous descent operations," Transportation Research Part C: Emerging Technologies, vol. 85, 2017. 